\title{
Cancers bronchiques : des nouveautés à affiner pour la routine
}

\author{
Lung Cancer: New Routine Methods to Be Refined
}

\author{
G. Robinet $\cdot$ A. Vergnenegre \\ (C) Lavoisier SAS 2016
}

La prise en charge des cancers bronchiques non à petites cellules s'est considérablement modifiée ces dix dernières années, bénéficiant des progrès obtenus dans les domaines diagnostiques et thérapeutiques. Et ces progrès se sont traduits par des gains de survie remarquables pour certains sous-groupes de patients.

Même si la lutte contre le tabagisme reste et doit rester notre préoccupation principale en matière de prévention, les données récentes sur l'intérêt d'un dépistage pour les populations à risque [1] orientent vers une baisse de mortalité. Les modalités de mise en place d'un tel dépistage en France restent encore débattues (voir l'article de C. Chouaid [2]).

Mais c'est surtout dans le domaine des thérapeutiques médicamenteuses que les progrès ont été les plus marqués. Et chaque avancée significative ouvre des perspectives nouvelles, toujours portées par une perpétuelle quête de guérison (ou de rémission complète, pour les spécialistes plus réalistes). Les exemples ne manquent pas, les histoires des mutations de l'EGFR et des translocations ALK sont particulièrement représentatives.

Les TKI anti-EGFR de première génération ont permis un gain de survie sans progression et une qualité de vie sous traitement nettement améliorée par rapport à la chimiothérapie classique, pour les patients mutés EGFR [3]. Mais sans pour autant obtenir une guérison, car après la période de rémission, la résistance au traitement s'installe (mutation T790M dans près de $50 \%$ des cas). L'étape suivante comporte maintenant des TKI de troisième génération, actifs sur la mutation de résistance, permettant une période de rémission supplémentaire... avant probablement la reprise évolutive. Encore une étape à franchir. La course reste acceptable pour les patients tant qu'elle offre un espoir de rémission dans des conditions de vie satisfaisantes, ce qui est le cas

\footnotetext{
G. Robinet $(\bowtie)$

Institut de cancérologie, CHU Morvan, F-29200 Brest, France

e-mail : gilles.robinet@chu-brest.fr
}

\section{A. Vergnenegre $(\square)$}

Service de pneumologie, CHU de Limoges,

F-87000 Limoges, France

e-mail : alain.vergnenegre@unilim.fr avec la majorité des TKI. Le revers de la médaille est la nécessité de mettre en évidence le mécanisme de résistance imposant un nouveau prélèvement tumoral : rebiopsie parfois invasive pour le patient, peut-être un jour avantageusement remplacée par la recherche d'ADN circulant, lorsque la sensibilité de l'examen sera améliorée (voir l'article de J.B. Auliac [4]).

Le développement récent de l'immunothérapie (inhibiteurs des points de contrôle anti-PD1, anti-PDL1 ou antiCTLA4) est un des progrès thérapeutiques les plus récents. Comme d'autres cancers, le NAPC bénéficie de l'apport de cette thérapeutique nouvelle (cf. article de C. Gauvin [5]). Un gain en survie globale est constaté, tout en préservant la qualité de vie sous traitement par rapport aux chimiothérapies classiques. Même si l'efficacité de l'immunothérapie ne semble concerner qu'un faible nombre de patients (taux de RO environ $20 \%$ ), elle semble durable (courbe de survie en « plateau » avec un taux de survie d'environ $20 \%$ à deux ans) lorsqu'elle est administrée en seconde ligne pour des NAPC métastatiques [6]. Mais contrairement au développement des TKI de l'EGFR ou ALK, thérapeutique ciblée sur l'anomalie mutationnelle, il n'existe pas à ce jour un marqueur prédictif discriminant d'efficacité permettant de définir avec une précision raisonnable les groupes de patients susceptibles d'en bénéficier de ceux qui a contrario ont un risque de perte de chance en recevant l'immunothérapie par rapport à la chimiothérapie. Pourtant, le développement des molécules va bon train, saine compétition entre les acteurs de l'industrie pharmaceutique, dont on espère que les grands bénéficiaires seront les patients et les systèmes de santé. Il s'agit de prouver l'efficacité de l'immunothérapie en première ligne seule (anti-PD1 ou anti-PDL1) ou en combinaison avec un anti-CTLA4 ou une chimiothérapie classique. Les essais sont pour la plupart soit en cours de recrutement, soit en cours d'analyse. L'étape suivante consistera à redéfinir la stratégie de prise en charge des NAPC à tous les stades en y intégrant l'immunothérapie, en précisant les séquences thérapeutiques. La recherche académique y prendra sa place. Espérons qu'avant cela un biomarqueur prédictif plus spécifique aura été précisé. 
L'apport des traitements antiangiogéniques dans la prise en charge des NAPC a été également remis au goût du jour par l'arrivée de nouveaux produits (anticorps et TKI), mais un peu effacé par le buzz médiatique de l'immunothérapie (anti-PD1 et PDL1), dont les gains en survie paraissent supérieurs. Une fois les stratégies thérapeutiques de prise en charge mieux définies, on peut penser que ces molécules retrouveront une place justifiée en association en deuxième ou troisième ligne (cf. article de E. Dansin [7]).

Mais les progrès thérapeutiques concernent également les modalités de radiothérapie, avec notamment le recours de plus en plus fréquent à la stéréotaxie, à tous les stades de la maladie. À l'évidence, cela conforte d'autant plus le rôle primordial des réunions de concertation pluridisciplinaire où l'avis de toutes les disciplines peut être requis pour tous les stades. Mais bien évidemment, les stades localement avancés sont ceux pour lesquels les traitements combinés sont requis (cf. article d'I. Lafay [8]). Les essais thérapeutiques sont plus difficiles à mener du fait du plus faible nombre de patients éligibles, mais le GFPC poursuit ses recherches dans ces stades, fort de son expertise, et propose un essai (GFPC 01.14) évaluant la faisabilité et l'efficacité d'une radiothérapie classique associée à une radiothérapie stéréotaxique en combinaison avec la chimiothérapie pour des NAPC de stades localement avancés avec une tumeur primitive périphérique.

Cette mise au point que la revue Oncologie a demandée au GFPC ne pouvait pas couvrir l'ensemble des aspects de la prise en charge des cancers bronchiques. En tenant compte des données nouvelles et des domaines d'expertise du groupe, nous l'avons complétée par des focus sur des situations cliniques particulières :

- les sujets âgés représentent une part importante des patients atteints de cancers bronchiques. La validation d'échelles permettant d'optimiser leur prise en charge est toujours d'actualité [9], l'article ci-après de Corre et al. [10] y fait logiquement allusion. L'intérêt de l'immunothérapie en première ligne pour des patients âgés fragilisés sera étudié dans l'essai eNERGY (GFPC 08.2015);

- les métastases cérébrales des cancers bronchiques (cf. article d'I. Monnet [11]) sont également un sujet sur lequel le GFPC travaille depuis plus de 20 ans [12]. L'actualité aussi bien par l'apport des nouvelles techniques de radiothérapie que par l'arrivée des thérapies ciblées impose de revoir les arbres décisionnels afin d'aider la discussion lors des RCP. La complexité croissante liée aux situations multiples justifie la mise en place dans certains centres d'une RCP dédiée pour les métastases cérébrales ;

- le mésothéliome reste une maladie rare, et les pics d'incidence prévus ne semblent pas avoir été atteints. Du fait de sa rareté, les mises au point en sont d'autant plus justifiées (cf. l'article de L. Greillier [13]). L'étude française à laquelle le GFPC a très largement contribué ne représente qu'un aspect de la prise en charge des mésothéliomes qui bénéfieront peut-être eux aussi des nouvelles thérapeutiques, et notamment des immunothérapies ou des thérapies vaccinales (essais en cours).

En espérant que cette mise au point répondra à vos attentes.

Liens d'intérêts : A. Vergnenegre - Conseil et expertise pour : Roche, BMS, AstraZeneca, Lilly, MSD.

\section{Références}

1. Aberle DR, DeMello S, Berg CD, et al (2013) Results of the two incidence screenings in the National Lung Screening Trial. N Engl J Med 369:920-31

2. Chouaid C, Rousseau-Bussac G, Jabot L, et al (2016) Le dépistage du cancer bronchopulmonaire par tomodensitométrie thoracique à faible dose en France : enjeux et perspectives. Oncologie 18:392-96

3. Masters GA, Temin S, Azzoli CG, et al (2015) Systemic therapy for stage IV non-small-cell lung cancer: American Society of Clinical Oncology Clinical Practice Guideline update. J Clin Oncol 33:3488-515

4. Auliac JB (2016) Les cancers du poumon avec une mutation oncogénique : une prise en charge de plus en plus spécifique. Oncologie 18:371-75

5. Gauvain C, Lena H, Corre R, et al (2016) Immunothérapie : un nouveau paradigme dans la prise en charge du cancer bronchique non à petites cellules. Oncologie 18:365-70

6. Borghaei H, Paz-Ares L, Horn L, et al (2015) Nivolumab versus docetaxel in advanced nonsquamous non-small-cell lung cancer. N Engl J Med 373:1627-39

7. Lauridant G, Kotecki N, Pannier D, Dansin E (2016) Place des antiangiogéniques dans le traitement des cancers du poumon. Oncologie 18:409-18

8. Martel-Lafay I (2016) Radiothérapie et traitements combinés des cancers bronchiques non à petites cellules localement avancés. Oncologie 18:385-91

9. Corre R, Greillier L, Le Caër H, et al (2016) Use of a comprehensive geriatric assessment for the management of elderly patients with advanced non-small-cell lung cancer: the phase III Randomized ESOGIA-GFPC-GECP 08-02 Study. J Clin Oncol 34:1476-83

10. Corre R, Le Caer H (2016) Spécificités de la prise en charge du CBNPC chez les sujets âgés. Oncologie 18:401-8

11. Monnet I (2016) Prise en charge des métastases cérébrales des cancers du poumon. Oncologie 18:376-84

12. Barlesi F, Gervais R, Lena H, et al (2011) Pemetrexed and cisplatin as first-line chemotherapy for advanced non-small-cell lung cancer (NSCLC) with asymptomatic inoperable brain metastases: a multicenter phase II trial (GFPC 07-01). Ann Oncol 22:2466-70

13. Greillier L (2016) Le mésothéliome : maladie orpheline ? Oncologie 18:397-400 